\title{
El altruismo recíproco imposible de Stirner. El problema de la inasunción biológica en las Humanidades, III
}

\author{
Atafrascas Orbinio de la Estigia*
}

April 22, 2020

\begin{abstract}
Intellectual blindness for those who believe they have the fleece of legitimacy, is the fundamental reason for advising a rigorous, scientifically based epistemology of human relations, including, or exceedingly, those contained in political theories, whose object is mere superstition, as is known. Stirner's infrequent political position, individualistic or selfish anarchism, despite some of his accurate analyzes, does not avoid the same criticism; in essence, his plot adventures are empty statements, linguistic games, lack of clarity, the same idealistic dogmatism that constantly attacks, poetizes everywhere, and, even basing his cause on nothing, he forgets that he is a living being. Is that nothing?
\end{abstract}

\section{Resumen}

La ceguera intelectual para quien cree poseer el vellocino de la legitimidad, es la razón primordial para aconsejar una epistemología rigurosa con base científica de las relaciones humanas, incluyendo, o sobremanera, las encerradas en las teorías políticas cuyo objeto es, como es conocido, mera superstición. La infrecuente posición política de Stirner, el anarquismo individualista o egoísta, pese a algunos de sus análisis certeros, no evita idéntica crítica; en esencia, sus peripecias argumentales son afirmaciones vacías, juegos lingüísticos, falta de claridad, constantemente el mismo dogmatismo idealista del que procede y ataca, poetiza por doquier, y, aun basando su causa sobre nada se olvida que él es un ser vivo ¿Es eso nada?

\section{Introducción}

A lo largo de la historia el hombre ha ensayado un buen número de formas de gobierno, esto es, de organizaciones jerárquicas o estructuras diversas que permiten manejar el poder político y teniendo a éste a su vez conformado con determinadas representaciones o cargos henchidos con unas tareas o funciones establecidas por unas normas ad hoc o previas. Sin llegar hasta el presente, son suficientes las muestras de distintos sistemas en los textos clásicos de Heródoto

\footnotetext{
${ }^{*}$ Facultad de Filosofía A Fraja, Camiño Fondo, 7. Email: ng110777@gmail.com
} 
y Tucídides ${ }^{1}$. La estabilidad y el derrocamiento de tales sistemas políticos ha ocupado a todos los pensadores de la política (Linz, 1978: 3 [21]). Los ensayos, algunos conscientemente formulados, se han aplicado en comunidades con un cierto grado de organización y complejidad, básicamente en comunidades con historia escrita; y a menudo con resultados consabidos, como los de Platón. Siempre se ha descartado en los grupos humanos, por pequeños que fuesen, la abolición de cualquier norma. Estas se antojan esenciales para reglar la conducta de los miembros y que estos no acaben dominando unos sobre otros, discutiendo o agrediéndose de continuo.

Pero el camino sobre cómo gestionar las comunidades humanas ha transcurrido intelectualmente siempre en sentido inverso. De las ideas a las cosas. De lo que pienso, por loco que sea, a lo que debe ser. Sin importar el sujeto de partida, el Homo sapiens, vamos a diseñar un cierto tipo de sociedad que cumpla con las reglas que me acabo de inventar febrilmente y que se aplicarán hasta que yo lo diga, tipo Licurgo ¿Quiénes son los que se arrogan esas capacidades legislativas? Respecto de estos pensadores que escriben sobre cómo debe organizarse una sociedad, hay autores que aglutinan el interés de los hermeneutas porque disponen de unas características que los individualizan, un aura o una crítica injusta que los marcan para siempre, o una idiosincrasia pertinaz. En el campo de la filosofía política, Stirner es uno de estos que ha sido lacerado por casi todos y desde el principio por haber escrito un perverso y triste libro, sí, un infame trabajo (Lange, 1866: 435 19]), un libro famoso pero infame (Biermann, 1906: 52 [2]), un extraño libro (Voländer, 1911: 462 [35]), y con toda probabilidad y para muchos autores el más extremo de los descendientes intelectuales del hegelianismo. No obstante, acaso las peores críticas provengan del extenso libelo de Marx \& Engels (Marx \& Engels, 1974: 532 [25]), que aciertan en ocasiones pero sólo por el hecho de envolver la ansiada eliminación de Bakunin en la destructiva crítica a Stirner bajo la sospecha realista de que las ínfulas anarquistas de aquél se nutrían en parte de éste y era necesario depurar al ruso por medios indirectos (Paterson, 1971: 128 [28]; Calasso, 2014: 22-23 [5]). O bien, como sostiene Derrida, Marx no quería un 'doble', un 'mal hermano', un 'enemigo', una imagen especular que te pregunta 'pero ese otro, ¿qué es?' (D'Angelo, 2018: [7]); había que borrar a Stirner tildándolo de ser el mayor idealista. O ambas cosas y alguna más.

En cualquier caso, nunca ha sido fácil para la historiografía filosófica adscribir a Stirner un lugar definido en la Historia de la Filosofía (Paterson, 1971: 126 [28]). En Grecia solía decirse que no puede decirse de un hombre que su vida haya sido afortunada hasta que haya muerto, hasta su final. No parece el caso de Stirner que, a las penurias comunes a todos, hemos de añadir la muerte de su primera mujer y su hijo en el parto, la pérdida o abandono del empleo como profesor de señoritas tras la publicación de su libro, el divorcio de su segunda mujer tras haberle, él, dilapidado su fortuna, la enfermedad, las deudas, la prisión y la miseria de sus últimos años (Mackay, 2005: 179-217 [23] Leopold, 2011: 21-41 20]). El abúlico como Amiel, odiaba la política, temía y odiaba el socialismo, un solitario por naturaleza (Huneker, 1907: 332-333 [16]). El egoísta insobornable, el aristócrata de la moral, el rebelde contra todo, el defensor del yo, del único, muere enfermo, solo, vilipendiado y por fin olvidado a los 49 años.

\footnotetext{
${ }^{1}$ Puede leerse con provecho una interpretación resumida en Grote (2001 [15])
} 
Se acabó la solicitada 'especie de derecho de domicilio'. Su anhelada asociación de egoístas, por fin, se consuma en el cementerio. Cada uno para sí.

\section{Metodología}

En la medida de lo posible, no utilizaré los habituales énfasis stirnerianos para no sobrecargar la lectura, salvo cuando quiera recalcar su egoísmo pseudoapodíctico. Para la referencia del texto stirneriano utilizaré ad libitum una versión inglesa, o bien otra española. Los pasos que seguiré son los siguientes:

(i) Exposición de un párrafo de Stirner. Podría haber elegido de entre un abultado elenco, pero me decanto por éste, casi al azar, que resume la futesa y huera causa en la que basó su discurso.

(ii) Causas que están en la raíz de sus expresiones y consecuencias que se derivan del párrafo stirneriano.

(iii) Dos palabras sobre el anarquismo de Stirner.

(iv) Conclusiones.

(v) Agradecimientos.

\section{$3 \quad$ El hecho}

Stirner, escribe en 'The Ego and Its Own':

"The case stands otherwise when what is yours is not made into something that is of itself, not personified, not made independent as a 'spirit' to itself. Your thinking has for a presupposition not 'thinking', but you. But thus you do presuppose yourself after all? Yes, but not for myself, but for my thinking. Before my thinking, there is I . From this it follows that my thinking is not preceded by a thought, or that my thinking is without a 'presupposition'. For the presupposition which I am for my thinking is not one made by thinking, no one thought of, but it is posited thinking itself, it is the owner of the thought, and proves only that thinking is nothing more than property, that an 'independent' thinking, a 'thinking spirit', does not exist at all."

(Stirner, 2000: 357 [31] pág 437 en la española [30])

\subsection{Causas y Consecuencias}

Muchos están de acuerdo en que el libro de Stirner, es uno de los más subversivos; un texto radical y extremo, mal leído, mal interpretado y peor comprendido pese a su sencillez (McQuinn, 2012: 3 y ss. 24]). No es cierto, pero voy a suponer que sí. De acuerdo, entonces, ¿de qué está hablando Stirner? Casi todos coinciden en que el asunto del individuo y su egoísmo, el ego, el único y su propiedad, son los conceptos clave que aborda Stirner ¿De qué forma lo hace? Pues obviamente, escribir sobre tales términos puede llevarnos a muy diferentes 
lugares dependiendo de nuestras intenciones o ideología. Cualquiera puede observar que el texto stirneriano que cito es un galimatías, que me abstengo de desmenuzar en detalle, y, en general, su libro ha recibido incontables varapalos. A las famosas, primerizas y contestadas críticas al contenido de Szeliga, Feuerbach y Hess (Stirner, 2012 32]), en cierta medida por la notoria ausencia de moral en Stirner o a la pretensión de eliminar cualquier tipo de moralidad, añadamos la redacción abstrusa y los evidentes 'efectos soporíferos' de las 'belicosas fanfarrias' (Marx \& Engels, 1974: 532 [25]).

Stirner habla de sí mismo, de su ego, del yo, de su yo como una entidad no nouménica cognoscible; sin embargo, Stirner no conoce su única propiedad, su mentada inmanencia, ni cómo se relaciona causalmente con el resto de objetos o seres, ni cómo está compuesto, ni cómo funciona. Puede negar que haya esencias, o bien ousías; aceptamos con naturalidad el realismo epistemológico, la existencia de 'una explicación satisfactoria de cómo se puede afirmar que hay una correspondencia o parecido entre algunas de nuestras creencias y la realidad, dado que no tenemos un acceso independiente a dicha realidad' (Diéguez, 2008: 68 [9]), pero, ¿y los detalles?, ¿cómo se atreve a afirmar quién es? Ni siquiera hoy, una persona sana podría indicarnos la morfología de su coronaria o el lumen de su tortuoso conducto cístico y todos sabemos que ambos hechos son exactamente cruciales para muchas personas. No sabe cómo está construido, ni cómo funciona, ni quién es realmente y no obstante insiste en asertos que no son biológicos o ecológicos aunque se fundan y están construidos sobre una entidad biológica; sólo retórica plúmbea, su fantasma de los fantasmas, un narcótico de la razón. Nos encontramos, como tan a menudo, con el argumento más falaz de todos, de cómo un sujeto se conoce a sí mismo como objeto epistemológico, de sostener que sólo existe el yo, su yo, y el resto de pensadores anda errado con conceptos supranaturales y sustancias filosóficas; los otros no conocen o no se percatan o temen las consecuencias de la existencia del único y su propiedad, la abolición de toda ley moral y de toda ley en general; pero Stirner sí. Él sí. Él ha resbalado en un acantilado y en vez de agarrarse se ha dejado caer en la grieta de los percebes y bálanos, sin daño aparente. Por coherencia. Pero mal entendida. Si es claro que comete el mismo error de que todo está mal, salvo lo mío, lo peor es que lo suyo no admite un análisis somero. Habremos de concluir que incurre en lo que Nietzsche dijo de los filósofos: su falta de sentido histórico es el defecto hereditario en los análisis introspectivos sobre el hombre (Nietzsche, 2000: 16 [26]), y es el caso del yo metafísico stirneriano.

Pero además, y fundamentalmente, Stirner no se da cuenta, o lo ignora a sabiendas, de que nada tiene sentido si no existe un alter ego. No existiría él, ni nadie más. Ni uno solo de sus antepasados directos murió virgen. Su abulia y su odio a las revoluciones y movimientos de carácter socialista, incluso aún suponiéndolos contrarios y certeros, lo confundieron y lo llevaron a eliminar cualquier sustento para un discurso coherente. El extremismo no le importó, pues lo diferenciaba. Su misantropía no lo llevó al suicidio, vaya, y sus diatribas lo colocaron en una situación extrema incoherente. Si él es el único, su único propietario, el que tiene el poder, si no hay nada que no pueda agenciarse como suyo sin trabas legales o morales, ¿por qué no lo hace? Porque no puede. No tiene el poder, no tiene la fuerza necesaria, ni siquiera la concha para enroscarse en su interior. Todos los alegatos stirnerianos, si bien sostenidos aquí y allá 
por algunos argumentos rayanos en la sensatez, son una sarta de letanías para una reata de conversos. Se le ha criticado, y yo mismo con estas letras, pero si filosóficamente podríamos extendernos decenas de páginas, biológicamente no hay caso. Stirner no es un paramecio que se valga por sí mismo y se reproduzca por fisión binaria.

El analfabetismo científico de todo ese tropel de hegelianos, aunque no sólo ellos y Stirner, su desconocimiento de Malpighi o de la teoría celular de Schleiden \& Schwann, demuestran su inepcia, su indigencia intelectual, su incapacidad para pensar comme il faut respecto al hombre como especie. A la par, su exaltación del hombre metafísicamente construido, con cualquier tipo de énfasis, sus pedestales para sus propios términos, la entronización y reificación de sus memeces axiológicas que en no pocas ocasiones han terminado en muerte y sufrimiento de aquél a quien en origen se pretende defender, su abuso miope y subjetivista del post hoc ergo propter hoc, son la prueba de la incomprensión de lo que es el caso y de los efectos que esas causas producirán, la inopia del pardillo que ya no lleva sotana pero conserva el idealismo típico con la tonsura o la marca del alzacuellos tapada por la barba.

¿Por qué el título dice 'altruismo recíproco imposible'? Porque el altruismo recíproco humano se da en multitud de contextos con una enorme diversidad de circunstancias ambientales y consecuencias personales, en realidad, siempre que 'the benefits of reciprocity depend on the unequal cost/benefit ratio of the altruistic act' (Trivers, 1971: 36 34); en nuestro caso, el caso de Stirner, hemos de suponer cabalmente que este autor escribe un libro con el objetivo de 'sharing knowledge' (Trivers, 1971: 36 34]), esto es, de enseñar aquello que ha descubierto a los que lo ignoran. Es el común juego intelectual de los que no pueden demostrar fehacientemente lo que afirman. No es posible reproducir el trabajo de Stirner, es suyo, nada más. Ahora bien, el objeto del libro es mostrar, enseñar, que no hay moral, ni ley, ni Dios, ni autoridad, etc., es decir, que no es posible el altruismo recíproco, ningún tipo de altruismo pues hemos de ser solipsistas pseudo-racionales. En otras palabras, por muy iracundo que uno se ponga contra algún tipo de estructura intelectual, si ésta se refiere, denota a una cierta parcela de la realidad, entonces hay que andar con cautela so pena de incurrir en contradicciones o en falacias non sequitur ¿Cómo referirse de continuo al 'yo' como único, si hay otros que mediatizan mi conducta y de los que dependo? ¿Cómo destruirlo todo esperando que permanezca una torre de marfil? Obviamente, 'no concept of group advantage is necessary to explain the function of human altruistic behavior' (Trivers, 1971: 48 34]), pero por ello mismo, tal concepto carecería de sentido si no hablásemos de una especie social ¿A dónde conducen los desvarios stirnerianos? A la contracción de todo en un yo que no puede darse.

Pero, el libro de Stirner admite otras interpretaciones menos cáusticas aunque se diga que 'el individualismo estirneriano es considerado la plasmación del egoísmo supremo, incompatible con cualquier tipo de vida social; y su práctica comportaría la ley de la selva, un infierno hobbesiano de guerra civil de todos contra todos, una liberación del yo terriblemente antisolidario, un yo a la búsqueda exclusiva de la satisfacción egoísta, del yo material, sin obligaciones morales ni sociales' (Díez, 2007: 126 [10]). Pero si las ideas stirnerianas conducen al culto del yo, al de mi propio ego y eso es inmoral porque desaparecen 
la justicia social, la fraternidad o la solidaridad y se ampara cierto movimiento ilegalista, ¿qué le pueden importar a Stirner las críticas éticas si de eso se trata, de acabar con la autoridad, con las clásicas ideas absolutas o supremas? Sólo quiere una asociación de egoístas en el que cada uno se apropie de su cuerpo y busque $s u$ libertad sin que ni aquél ni ésta dependan de gobiernos, dioses o Ideas platónicas. Quizá simple, quizás imposible, pero inapelable. La superioridad de su moral aristocrática cercenando cualquier atisbo ético, no se ha desarrollado de la forma esperable por muchos en el seno del movimiento anarquista por las consecuencias penales obvias y tal vez porque su desconexión con la biología humana es evidente.

¿Qué se sigue del Único? que es una unidad que 'mayor no puede pensarse ${ }^{2}$ y por ello mismo y respecto del resto de Únicos es tan diferente que 'mayor no puede pensarse'. Así que soy único y no hay nadie como yo. E idéntico razonamiento hacen los demás. Pero este solipsismo dulcificado por lo certero de tener un yo pero haber otros, se vuelve simple metafísica solipsista empalagosa cuando el resto son objetos fenoménicos acaso estudiables pero también ellos y sus propiedades contingentes para el yo stirneriano (Para otra visión, Schiereck, 2015 [29]). Si ya nos obnubila la razón el extenso, abstruso y perverso racionalismo solipsista de una teología de la Trinidad (por ejemplo, Greshake, 2001 [14]), la teología de la unicidad stirneriana buscando lo mismo, nos fastidia con su infantilismo, con su querer pero no poder. De una perikhóresis como unidad diferenciada y no una exclusiva yuxtaposición de elementos diversos insolubles entre ellos, a la demolición de la circuminsessio de Aquino, la erradicación fulminante de la relación mutua de todos los hombres.

'Everything is carried out in language' (Wittgenstein, 2005: 279e-286e [36]); si todo se gesta en el lenguaje, nada más claro para Stirner, todo parece radicar en un problema con los fantasmas del pasado, con seres absolutos, espíritus, esencias, sustancias o sustantivos con mayúscula. Sin embargo, semeja que su análisis subsiste para su mismo caso y, como era de esperar, con su mismo diagnóstico y solución; el 'fantasma de los fantasmas', está repleto de comillas, de cursivas, de mayúsculas y otros énfasis ¿Acaso lo que necesitábamos era sustituir unos entes como 'Dios' o el 'Hombre' por los de 'Yo' o 'Único'? El camino que lleva a Stirner al ateísmo y al materialismo, su meridiana conclusión, ya había sido transitado por otros antes, como Holbach o La Mettrie, pero el materialismo de estos ya había también sido rechazado por unanimidad por los filósofos (Finger, 1961: 44 [12]); si bien, estos autores no llegaron tan lejos como Stirner que incluso llamando fanáticos a los pensadores más cultos, habría rechazado absolutamente una etocracia al estilo de Holbach (Stirner, 2000: 44 y nota 53 [31). De nuevo el problema de la amoralidad como un hecho inmoral de Stirner.

El problema en que está envuelto es que ensimismado en su descubrimiento del ego, ha confundido categorías inmiscibles; llevado por el ardor de una idea feliz, enfrascado en ella, la generaliza con fruición y sin criterio; que si alguna parte de sus ataques a la legitimidad o autoridad de gobiernos y gobernantes, su negación de la existencia de leyes morales y otras layas y recalcitrante insistencia en la importancia de uno mismo, suenan verosímiles e incluso correctas, su

\footnotetext{
2'Quod maior sit qua[m] cogitari possit'. Recordamos aquí el Proslogion de San Anselmo (Por ejemplo, en Logan, 2009: 25 22])
} 
extrapolación a terrenos menos nomológicos es arriesgada, errónea de hecho.

\subsection{Dos palabras sobre el anarquismo de Stirner}

El papel de Stirner en los movimientos anarquistas 'ha sido siempre más decorativa que real' (Paterson, 1971: 132 [28]). Y eso teniendo en cuenta que, con generalidad, los anarquismos son mucho más complejos de lo que habitualmente se reconoce (Cornell, 2016, 'Conclusion' 6]). La anarquía nunca ha sido bien vista porque la mayoría ve inviable o inconcebible esta opción individualista, o bien prefiere mantener la estasis individual y colectiva con normas que coadyuven al funcionamiento económico, político y social idóneo (Nozick, 1988 [27]) En caso contrario, podemos observar en distintos ámbitos la influencia anarquista individualista traducida en locuras que van desde el pensamiento simplemente extraviado hasta, sin exclusión, la acción abominable (Kaczynski, 2008: 38 [17]).

Así, Nozick pretende desmontar al anarquista individualista, aunque no cita ni una sola vez a Stirner en su libro 'Anarquía, Estado y utopía' ¿Por qué lo hace? Pues, porque la teoría anarquista, si es sostenible, socava todo el objeto de la filosofía política' (Nozick, 1988: 17 [27]). Para Nozick, el estado ultramínimo se formaría de manera natural en cuanto un puñado de personas se agrupasen para obtener un mayor beneficio. Se produciría por un sistema de mano invisible. Y consiguientemente, la asunción de la protección individual y la redistribución de los recursos surgirían de forma espontánea y legítimamente moral. El uso coercitivo de la fuerza y la coacción para repartir bienes entre otras personas es el resultado, moral, de pasar de un estado ultramínimo a otro mínimo. Empero, Nozick ya considera inmoral, 'ni legítimo, ni justificable', un estado de mayor entidad que el mínimo (Nozick, 1988: 62 [27]). Cualquier anarquista, Stirner u otro, se llevaría las manos a la cabeza con semejante dislate. Pues, ¿qué significa en este contexto, 'más' o 'mayor'? Detalles, por favor ¿Quién, tras una epifanía politica y con poder, no colocaría donde le pluguiese el umbral de la moralidad o legitimidad? ¿A quién con el poder suficiente no le gustaría encarcelar a sus ciudadanos mientras nos susurrase al oído que es por nuestro interés, por nuestro bien, como un padre maneja a un niño pequeño? Stirner y el anarquista genérico tienen razón absolutamente. El Estado conculca los derechos del individuo, no hay más. Es intrínsecamente inmoral. Y para evitar ahora el problema conceptual, serio, de los derechos, diremos que el Estado, al menos, se arroga la determinación de modificar o eliminar al único y su propiedad por medio de leyes o la fuerza. Se dirá que no hay solución y que los argumentos del anarquista son correctos pero pírricos y que de todas maneras hay que actuar. Sea; pero ello no cambiaría la honestidad de algunos argumentos stirnerianos en detrimento de una filosofía política hipócrita y de una praxis execrable.

Aunque algún autor disiente (Paterson, 1971: 132 [28]), respecto de una seria contribución efectiva a la historia del anarquismo, no puede extrañar que Stirner sea considerado un adalid del anarquismo individualista heterodoxo, una 'black sheep' (Kinna, 2011: 42 y ss. 18), cuando irónicamente señala que 'se pide a los Estados que pongan fin al pauperismo' al tiempo que le resulta obvio que 'el interés del Estado es ser rico él mismo; poco le da que Pedro sea el rico y Pablo pobre; igual querría que fuese Pablo el rico y Pedro el pobre'; y estos a veces son un simple trasunto del Estado manejando la propiedad ajena y estableciendo legislativamente que esa propiedad no es mía, no es nuestra, sino 
de aquél, 'propiedad del Estado sin ser nunca propiedad del Yo' (Stirner, 2014: 329 [30]). En definitiva y en la praxis política, incluyendo las democracias realmente existentes, no sólo de iure, Stirner 'argued that all political systems lead in practice to the authoritarian suppression of the individual ego' (Blackledge, 2012: 20 [3]).

Stirner es un destructor, empeñado en aniquilar todo concepto general o abstracto, en desconfiar de las revoluciones cargadas de buenas intenciones siempre dogmáticas o idealistas, en eliminar mitos, autoridades, gobiernos y estados, en aplicar un escepticismo radical en todo embate erudito, teórico o especulativo, en destruirlo todo y no dejar nada en pie (Diéguez, 2008: 330 [9]).

El lugar extremo de la dialéctica, la alienación de los torpes lugares comunes, el final de las reapropiaciones idealistas, la disolución en el nihilismo de todos los movimientos que parten de Hegel, a eso se enfrenta un lector de Stirner, al nihilismo del yo como verdad de la dialéctica (Deleuze, 1983: 183-186 [6]). Parodiando y contrariando a Ghosh respecto a Hegel, al tratar de espiritualizarse en el dominio de lo no Absoluto, la filosofía sufre una disminución de la identidad en contraste con el logro de la poesía especulativa (Ghosh, 2019: capítulo 1 [13]).

\subsection{Conclusiones}

La lectura del libro de Stirner depara al iluso la ilusión de haberse topado con un zafiro, al anarquista el obtener algunos argumentos prístinos inaplicables en contra de la autoridad, al marxista el ensimismado y delectable desprecio de barbuda raigambre, al liberal el temor a que de Stirner se siga la imposibilidad de la minarquía; el científico solo percibe manchas en el papel, simple retórica y deseos inconclusos, pero racionalmente, y en conjunto, puede alegarse de la obra de nuestro autor y de su contenido lo siguiente:

(i) El libro de Stirner es farragoso y poco coherente con una línea argumental fragmentaria. Da la sensación de que, ingenuamente, inaugura los movimientos de indignación ${ }^{3}$ contra la ontología de la tradición a través de una gnoseología infantil que ve la paja en el ojo ajeno pero no la viga en el propio. A la liberación teológica de la teología feuerbachiana, como señaló nuestro autor, oponemos análogamente el similia similubus curantur stirneriano ( $C f$. Feuerbach 1973: 233 [11]). Poca curación y ninguna etiología, pues hace afirmaciones ontológicas sin apoyo de ningún tipo, salvo su introspección. Esto es, psicologismo subjetivista enroscado en sus númenes. Cómo no habría de aplicáserle a Stirner la definición de Schlegel sobre la poesía del momento, 'Transzendentalpoesie that combines the traditional 'self-mirroring' of the lyrical poet with 'the transcendental raw materials...' (Bruns, 2006: 177 [4]).

(ii) A la pregunta de que se demuestre la existencia de una silla dada, podrá responderse a lo Hume, '¿silla, qué silla?' (Tasset, 2010: 161 [33]). Es decir, ¿yo?, ¿qué yo? Nada más que 'la elevación del yo al rango de un absoluto que trasciende toda estructura y toda forma de ser' (Baigorria,

\footnotetext{
${ }^{3} \mathrm{Cf}$. 'Moralistic aggression and indignation in humans was selected for in order to counteract the tendency of the altruist, in the absence of any reciprocity' (Trivers, 1971: 54 [34]). Y esto en los dos sentidos, el indignado Stirner que se ve menospreciado y los airados e irritados críticos, que no se lo toleran.
} 
2007: 13 [1]). El solipsismo contrariando los ritmos que manifiesta el dualismo del título. Stirner no especifica ni explica los conceptos que encuentra dados. Si no vale la pregunta '¿qué es el hombre?', ¿qué tipo de respuesta nos otorgaría el sustituir el 'qué' por el 'quién'?; sobre todo porque no sea que nos topemos con un oxímoron particularista. Como tantos otros se ha puesto a escribir sin saber apenas nada del objeto sobre el que escribe. Pero, ¿pretende solucionar el problema de los universales cambiando las categorías epistemológicas?

(iii) Postulando el reinado del egoísmo y deseando una 'asociación de egoístas', no se percata de que la búsqueda del beneficio propio pasa por su reproducción, esto es, por no ser egoísta del todo, por transmitir parte de lo que es hacia otros. No es sólo que el egoísta no piense en seres supremos o ideas sagradas y haya de sacrificar a estos su interés personal, sino servir únicamente a este último. Si buscase coherencia no escribiría un libro que otros leyesen, para decir en él que lo escribe por su propio interés (Stirner, 2014: 377 30]) Detállalo. ¿Cuál sería ese interés, sino alcanzar ciertas cotas de ámbito social, del tipo que fuesen, que deseaba (Feuerbach, 1973: 233 [11)? ¿Acaso se habría molestado en escribir un libro así si fuese realmente el único sobre la Tierra? Somos seres sociales ¿Qué sentido tendría? ¿Psicológico? ¿El egoísta de hoy habrá de preocuparse del egoísta de mañana? En resumen, la incapacidad para entender las redes causales y que Stirner es un ser vivo que pertenece a una especie con una historia filogenética.

(iv) Bien claro es que cualquier crítica al egoísmo de Stirner podría eliminarse por el mero expediente de rebatirla ad hoc sosteniendo que en cualquier caso el sujeto procura, aunque no lo parezca su propio bien y no otro ajeno. Pero una explicación basada en una falacia ad hoc, no la hace más creíble, sino menos. Y siempre errando, perdiendo de vista que hablamos de una entidad biológica.

\subsection{Agradecimientos}

A una escalera de madera que sube desde la cocina girando un par veces a la derecha y, tras ella al comienzo del pasillo a la izquierda, al estrecho y luminoso cuarto sils-mariano con aquella gran ventana, de marcos de madera pintada con el blanco de los años, que daba al este, al mar y a los desmanes del pensamiento. $\mathrm{Y}$ a su propietario.

\section{Bibliografía}

[1] Baigorria, Oswaldo. Prólogo. In, Armand, Émile. El anarquismo individualista. Lo que es, puede y vale. Utopía Libertaria, La Plata, Terramar, 11-13 pp., [1916] 2007.

[2] Biermann, Wilhelm Eduard. Anarchismus und Kommunismus: Sechs Volkshochschulvorträge. Verlag, Deichert. Verlagsort, Leipzig, 184 pp., 1906. Disponible en archive.org. Acceso el 16/04/2020. 
[3] Blackledge, Paul. Freedom and Democracy: Marxism, Anarchism and the Problem of Human Nature. In Libertarian Socialism. Politics in Black and Red. Edited by Alex Prichard, Ruth Kinna, Saku Pinta \& David Berry. Palgrave Macmillan, 17-34 pp., 2012.

[4] Bruns, Gerald L. On the anarchy of poetry and philosophy: a guide for the unruly. Fordham University Press, New York, 274 pp., 2006.

[5] Calasso, R. Acompañamiento a la lectura de Stirner. Prólogo in El Único y su propiedad. Editorial Sexto Piso, Madrid, 9-53 pp., 2014.

[6] Cornell, Andrew. Unruly Equality: U.S. Anarchism in the Twentieth Century. University of California Press, Oakland, California, 2016.

[7] D'Angelo, Valerio. 'San Max' releido. Una defensa de Stirner contra Marx. Revista Internacional de Pensamiento Político, I Época, Vol. 13: 235-255, 2018.

[8] Deleuze, Gilles. Nietzsche et la Philosophie. Presses Universitaires de France Paris, 232 pp., [1962] 1983.

[9] Diéguez, Antonio. ¿Usó Nietzsche el peor argumento del mundo? Una indagación sobre las bases evolucionistas del antirrealismo nietzscheano. Estudios Nietzsche, 8: 65-90 pp., 2008.

[10] Diez, Xavier. El anarquismo individualista en España (1923-1938). VIRUS editorial/Lallevir S.L., 376 pp., 2007.

[11] Feuerbach, Ludwig. 'L' essence du christianisme' dans son rapport a 'L'unique et sa propiété' (1845). In Manifestes philosophiques. Textes choisis (1839-1845). (Traducción de Louis Althusser). Presses Universitaires de France, Paris, 221-237 pp., 1973.

[12] Finger, Otto. Von der Materialität der Seele. Beitrag zur Geschichte des Materialismus und Atheismus im Deutschland der zweiten Hälfte des 18. Jahrhunderts. Akademie-Verlag, Berlin, 191 pp., 1961.

[13] Ghosh, Ranjan. The Agonizing Agon: Meditations on a Conjugality. In Philosophy and Poetry. Continental Perspectives. Edited by Ranjan Ghosh. Columbia University Press, New York, primer capítulo, 2019.

[14] Greshake, Gisbert. El Dios Uno y Trino. Una teología de la Trinidad. Biblioteca Herder, Empresa Editorial Herder, Barcelona, 710 pp., [1997] 2001.

[15] Grote, George. A history of Greece: from the time of Solon to 403 BC. Condensed and Edited by J.M. Mitchell and M.O.B. Caspari [with a new introduction by Paul Cartledge]. Routledge, London, 978 pp., [1846-1856] 2001.

[16] Huneker, James. Max Stirner. The Ego and His Own by Max Stirner and Steven T. Byington. The North American Review, Vol. 185(616): 332-337 pp., 1907. 
[17] Kaczynski, Theodore J. Technological Slavery. The collected writings of Theodore J. Kaczynski, a.k.a. 'The Unabomber'. Introduction by Dr. David Skrbina. Feral House, Port Townsend, 431 pp., 2008.

[18] Kinna, Ruth. The mirror of anarchy: the egoism of John Henry Mackay and Dora Marsden. In: Newman, S. (ed.). Max Stirner. Critical Explorations in Contemporary Political Thought. Basingstoke: Palgrave Macmillan, 42-66 pp., 2011. Disponible online aquí. Acceso el 15/04/2020.

[19] Lange, Friedrich Albert. Geschichte des Materialismus und Kritik seiner Bedeutung in der Gegenwart. Verlag von J. Baedeker, Iserlohn und Leipzig, 855 pp., [1866] 1887. Disponible en archive.org. Acceso el 16/04/2020.

[20] Leopold, David. A Solitary Life. In: Newman, S. (ed.). Max Stirner. Critical Explorations in Contemporary Political Thought. Basingstoke: Palgrave Macmillan, 21-41 pp., 2011.

[21] Linz Storch de Gracia, Juan J. The Breakdown of Democratic Regimes. Crisis, Breakdown $\&$ Reequilibration. The Johns Hopkins University Press, Baltimore, 130 pp., 1978.

[22] Logan, Ian. Reading Anselm's Proslogion. The History of Anselm's Argument and its Significance Today. Ashgate Publishing Limited, Surrey, 213 pp., 2009.

[23] Mackay, John Henry. Max Stirner. His Life and his Work. Peremptory Publications, Concord, California, 227 pp., [1898] 2005.

[24] McQuinn, Jason. Clarifying the Unique and Its Self-Creation: An introduction to 'Stirner's Critics' and 'The Philosophical Reactionaries'. In Stirner, Max. Stirner's Critics. Translated by Wolfi Landstreicher. Introduction by Jason McQuinn. LBC Books \& Cal Press. Columbia Alternative Library, Oakland, 2-45 pp., 2012.

[25] Marx, Carlos \& Engels, Federico. La Ideología Alemana. Crítica de la novísima filosofía alemana en las personas de sus representantes Feuerbach B. Bauer y Stirner y del socialismo alemán en las de sus diferentes profetas. Ediciones Pueblos Unidos \& Ediciones Grijalbo S.A., 751 pp., 1974.

[26] Nietzsche, Friedrich. Human All Too Human (I). A Book For Free Spirits. The Complete Works of Friedrich Nietzsche, 3. Translated, with an Afterword, by Gary Handwerk. Stanford University Press, Stanford, California, 388 pp., 2000.

[27] Nozick, Robert. Anarquía, Estado y utopía. Fondo de Cultura Económica, 330pp., 1991.

[28] Paterson, R.W.K. The Nihilistic Egoist Max Stirner. Oxford University Press for the University of Hull. London, 322 pp., 1971.

[29] Schiereck, Larry Alan. Max Stirner's Egoism and Nihilism. Thesis Presented to the Faculty of San Diego State University for the Degree of Master of Arts in Philosophy, 52 pp., [1981] 2015. 
[30] Stirner, Max. El Único y su propiedad. Editorial Sexto Piso, Madrid, 452 pp., 2014.

[31] Stirner, Max. The ego and its own. Cambridge Texts in the History of Political Thought, edited by David Leopold. Cambridge University Press, 386 pp., 2000.

[32] Stirner, Max. Stirner's Critics. Translated by Wolfi Landstreicher. Introduction by Jason McQuinn. LBC Books \& Cal Press. Columbia Alternative Library, Oakland, 128 pp., 2012.

[33] Tasset, José L. Ensayo de una historia humorística de la filosofía. In La sonrisa del sabio. Ensayos sobre humor y filosofía. Ballester, M. \& Ujaldón, E. (Eds.). Editorial Biblioteca Nueva. Ensayo. Madrid, 149-184 pp., 2010.

[34] Trivers, Robert L. The Evolution of Reciprocal Altruism. The Quarterly Review of Biology, Vol. 46(1): 35-57 pp., 1971.

[35] Vorländer, Karl. Geschichte der Philosophie. II Band. Philosophie der Neuzeit. Verlag der Dürr'schen Buchhandlung, Leipzig, 524 pp., 1911.

[36] Wittgenstein, Ludwig. The Big Typescript, TS 213. Edited and translated by C. Grant Luckhardt and Maximilian A.E. Aue. German-English scholar's ed. Blackwell Publishing Ltd., 1054 pp., 2005. 